Article

\title{
Preparation of Cationic MOFs with Mobile Anions by Anion Stripping to Remove 2,4-D from Water
}

\author{
Tao Chen, Cong Zhang, Yuemei Qin, Haiguan Yang, Peng Zhang and Fanggui Ye * (it) \\ State Key Laboratory for the Chemistry and Molecular Engineering of Medicinal Resources, \\ College of Chemistry and Pharmaceutical Science of Guangxi Normal University, Guilin 541004, China; \\ chenmaoen2015@163.com (T.C.); congzhangchem@163.com (C.Z.); qym0774@163.com (Y.Q.); \\ yanghaiguan135588@163.com (H.Y.); 15677330721@163.com (P.Z.) \\ * Correspondence: fangguiye@163.com; Tel.: +86-0773-5856-104; Fax: +86-0773-5832-294
}

Received: 3 July 2017; Accepted: 24 July 2017; Published: 30 July 2017

\begin{abstract}
A cationic porous framework with mobile anions (MIL-101(Cr)-Cl) was easily and successfully synthesized by utilizing the stronger affinity of $\mathrm{F}^{-}$to $\mathrm{Al}^{3+}$ than $\mathrm{Cr}^{3+}$ in the chargebalanced framework of MIL-101(Cr). The structure, morphology and porosity of MIL-101(Cr)-Cl were characterized. The obtained new materials retain the high surface area, good thermostability, and structure topology of MIL-101(Cr). With the mobile $\mathrm{Cl}^{-}$anion, MIL-101(Cr)-Cl can be used as an ion-exchange material for anionic organic pollutions. In this work, 2,4-dichlorophenoxyacetic acid (2,4-D) was used as a model to test the absorption performance of this new material. This new material exhibited improved adsorbability compared to that of the original metal-organic frameworks (MOFs). At the same time, this material also shows high anti-interference performance with changing solution $\mathrm{pH}$.
\end{abstract}

Keywords: adsorption; 2,4-dichlorophenoxyacetic acid; ion exchange; metal-organic frameworks

\section{Introduction}

Water pollution has become a global problem and is receiving significant attention, especially in developing countries. Pollutants exert a detrimental effect on the environment and human health, and hence, many research efforts have focused on removing pollutants from waste water. So far, various technologies have been used to remove pollutants, including photo-reduction or degradation [1], bio-degradation [2], ion exchange [3] and adsorption [4]. Among the technologies, ion exchange is one of the most eco-friendly and economical technologies, because most of the materials can be reused, this method has high sensitivity and selectivity, and the operation process is simple. The existing organic materials that can be used in ion exchange are sol-gel adsorbents [5] and polymer resins or membranes [6], which show rapid exchange kinetics and high ion-exchange capacity, but their low thermal and chemical stability limit their application in water purification. Although inorganic ion exchangers such as zeolites [7] and layered double hydroxides [8] have high thermal and chemical stability, their exchange kinetics and ion-exchange capacity are low. Therefore, developing new materials with high ion-exchange capacity and thermal and chemical stability is still a challenging task.

Metal-organic frameworks (MOFs), which are formed by using a pre-formed organic linker with a metal ion or cluster, are a class of supramolecular material. Owing to their unique structure and properties such as coordination with unsaturated metal sites, good thermal stability, high surface area, and adjustable pore size, as well as their ability to modify their pore surfaces, MOFs have been widely used in gas storage [9] and separation [10], catalysis [11], drug delivery [12], molecular sensing [13], and photoelectron chemistry and electro-chemistry [14]. MOFs have also been used for removing various hazardous substances from the environment. The adsorption mechanisms of most hazardous 
substances are based on acid-base interactions, $\pi$-complexation, H-bonding, and coordination with open metal sites. However, the adsorption capacity may be affected by operating conditions such as the $\mathrm{pH}$ or ionic strength. Some studies on charged MOFs materials [15-18] have been reported and some of these materials were used as adsorbents to remove pollutants from waste water using ion exchange.

2,4-Dichlorophenoxyacetic acid (2,4-D) was one of the most widely used acidic herbicides and plant growth regulators in the past three decades. However, it is considered to be a potential carcinogenic and teratogenic pollutant. Its low pKa (2.8) and large-scale application led to its accumulation in soils and environmental waters [19]. Therefore, eliminating 2,4-D from the environment is a necessary goal. So far, various technologies have been used for removing 2,4-D, including ozonation, adsorption, ion exchange, electrochemical technology, and biological processes [20-24]. Adsorption and ion exchange are often used to treat 2,4-D-contaminated water, but both have their own strengths and weaknesses. Jhung et al. [25] studied MOFs (MIL-53(Cr)) for the adsorptive removal of 2,4-D from water for the first time and with a high absorption capacity, but the adsorption capacity decreased with increasing $\mathrm{pH}$ and negligible adsorption was observed when $\mathrm{pH}>6$. Bhardwaj et al. [26] demonstrated that surfactant-modified tectosilicates (clinoptilolite and zeolite $\mathrm{Y}$ ) and phyllosilicates (bentonite and montmorillonite) can be used to remove 2,4-D from water based on ion exchange, although the adsorption capacities are lower and the adsorption capacity drops off slowly at high $\mathrm{pH}$.

In the present work, a cationic MOF (denoted as MIL-101(Cr)-Cl) was prepared by sequentially exchanging $\mathrm{F}^{-}$in original MIL-101(Cr) with $\mathrm{Cl}^{-}$. This preparation method is simple and does not require a cumbersome modification process. We can obtain this ion-exchange material, which has excellent performance by a simple hot $\mathrm{AlCl}_{3}$ solution treatment step. In addition, this cationic MOF has higher adsorptive capacity for 2,4-D than other materials, even at high $\mathrm{pH}$.

\section{Results and Discussion}

\subsection{Characterization of As-Prepared Materials}

The crystallinity of MIL-101(Cr) and MIL-101(Cr)-Cl were monitored by powder X-ray diffraction (PXRD). As indicated by the PXRD (Figure 1), the synthesized MIL-101(Cr) exhibited the same characteristic structure in consistence as the reported work [12]. After anion stripping, the material (MIL-101(Cr)-Cl) still remained highly crystalline. It means that MIL-101(Cr)-Cl may still retain some characteristics of MIL-101(Cr), such as porosity.

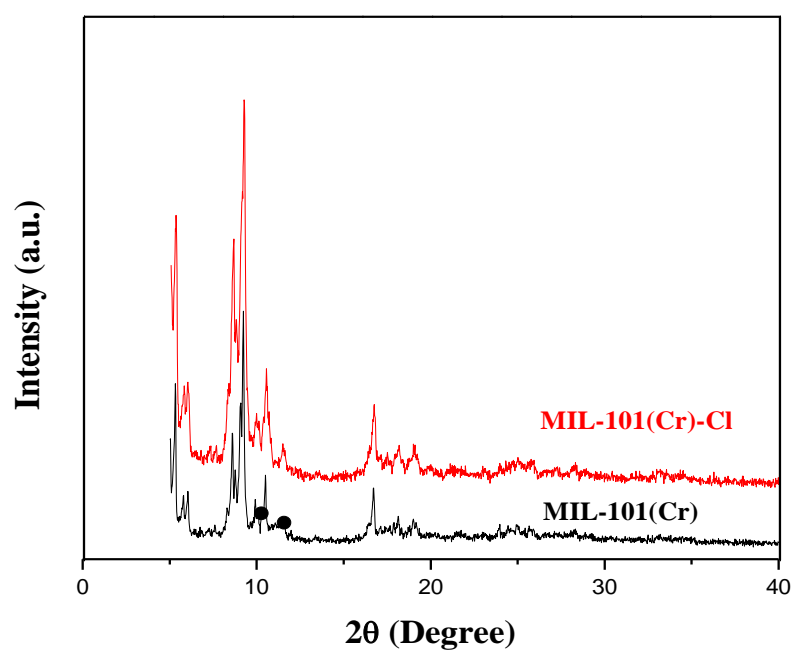

Figure 1. Powder X-ray diffraction (PXRD) patterns of MIL-101(Cr) and MIL-101(Cr)-Cl. 
To further characterize the porosity of the as-prepared materials, they were examined by nitrogen sorption. As shown in Figure 2, both samples have type-I isotherms with hysteresis loops, indicating a mesoporous structure. The $\mathrm{N}_{2}$ uptake of MIL-101(Cr)-Cl was much higher than that of the pretreated original MIL-101(Cr). The Brunauer-Emmett-Teller (BET) surface areas, pore volumes, and apertures are summarized in Table 1, and the pore size distribution is shown in Figures S1 and S2. The BET surface area and the pore volumes became larger after anion stripping. During the anion stripping procedure, $\mathrm{H}_{2} \mathrm{BDC}$ residues within the pores of $\mathrm{MIL}-101(\mathrm{Cr})$ were stripped of $\mathrm{F}^{-}$, and it is the reason why the BET surface area and the pore volumes became larger. The average pore size-which was the same as in the reported work [27]—remained the same, suggesting that the microvoids in MOFs did not collapse after anion stripping.

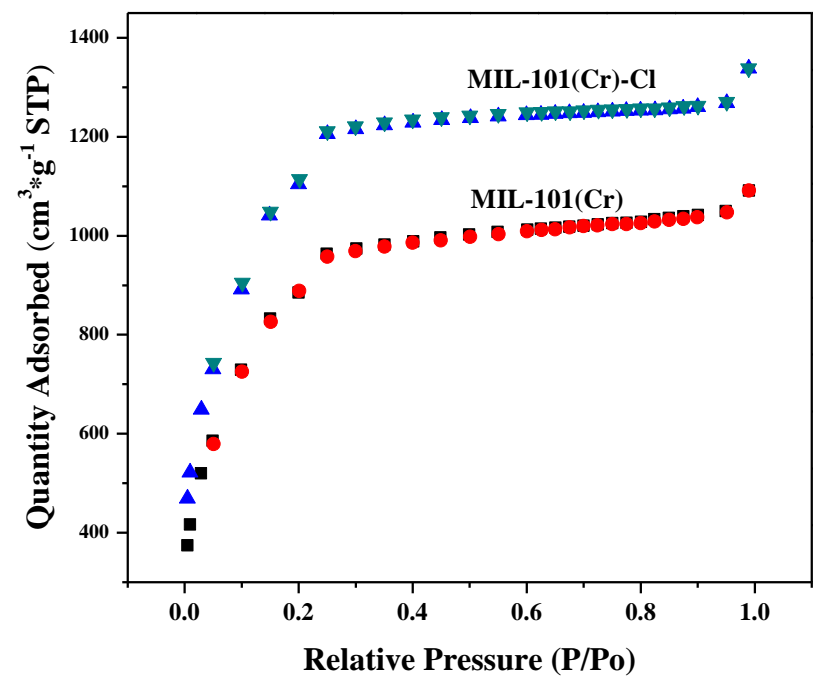

Figure 2. $\mathrm{N}_{2}$ adsorption-desorption isotherms of as-synthesized MIL-101(Cr) and MIL-101(Cr)-Cl.

Table 1. Some properties of MIL-101(Cr) and MIL-101(Cr)-Cl for $\mathrm{N}_{2}$ adsorption experiments at $77 \mathrm{~K}$.

\begin{tabular}{cccc}
\hline Sample & $\mathbf{S}_{\mathbf{B E T}}{ }^{\mathbf{a}}\left(\mathbf{m}^{2} / \mathbf{g}\right)$ & $\mathbf{V}_{\mathbf{p}}{ }^{\mathbf{b}}\left(\mathbf{c m}^{3} / \mathbf{g}\right)$ & $\mathbf{D}_{\mathbf{p}}{ }^{\mathbf{c}}(\mathbf{n m})$ \\
\hline MIL-101(Cr) & 3133.7 & 1.688 & 3.06 \\
MIL-101(Cr)-Cl & 3931.8 & 2.070 & 3.06 \\
\hline
\end{tabular}

a $\overline{\text { Brunauer-Emmett-Teller (BET) surface area; }{ }^{\mathrm{b}} \text { total pore volume; }{ }^{\mathrm{c}} \text { average pore diameter. }}$

The morphologies of MIL-101(Cr) and MIL-101(Cr)-Cl were investigated by Field Emission Scanning Electron Microscopy (SEM) (Figure 3). From Figure 3, it was apparent that the morphologies and size changed little after anion stripping. The sizes of these two materials were distributed homogeneously. This means that the SEM results agreed with the PXRD patterns and porosity results. Also, the Energy Dispersive Spectro-scopy (EDX) date showed that the $\mathrm{Cl}^{-}$content of MIL-101(Cr)-Cl was 10.58 wt \% (Table S1). The Thermo Gravimetric Analyzer (TGA) curves of MIL-101(Cr) and MIL-101(Cr)-Cl were obtained in air. As shown in Figure 4, the as-prepared materials had two weight loss steps. The first step was due to dehydrate of water that adsorbed within the pores of MIL-101(Cr), and the second step indicated that MIL-101(Cr) is stable up to $400{ }^{\circ} \mathrm{C}$. Therefore, the materials we prepared were typical MIL-101(Cr), and changed little after anion stripping. All the characterizations proved that MIL-101(Cr)-Cl retains the performance of MIL-101(Cr).

To demonstrate that $\mathrm{Cl}^{-}$has taken the place of $\mathrm{F}^{-}$and that $\mathrm{Cl}^{-}$can be removed, we performed some simple experiments [28]. One is the precipitation experiment performed before and after ion-exchange with $\mathrm{NaNO}_{3}$. Typically, $5 \mathrm{mg}$ of MIL-101(Cr)-Cl powders was soaked in $10 \mathrm{~mL}$ water or $80 \mathrm{mg} / \mathrm{mL} \mathrm{NaNO}_{3}$ for about $12 \mathrm{~h}$. After centrifuging, the upper liquid was taken out, and then $5 \mathrm{mM}$ $\mathrm{AgNO}_{3}$ solution was added. 

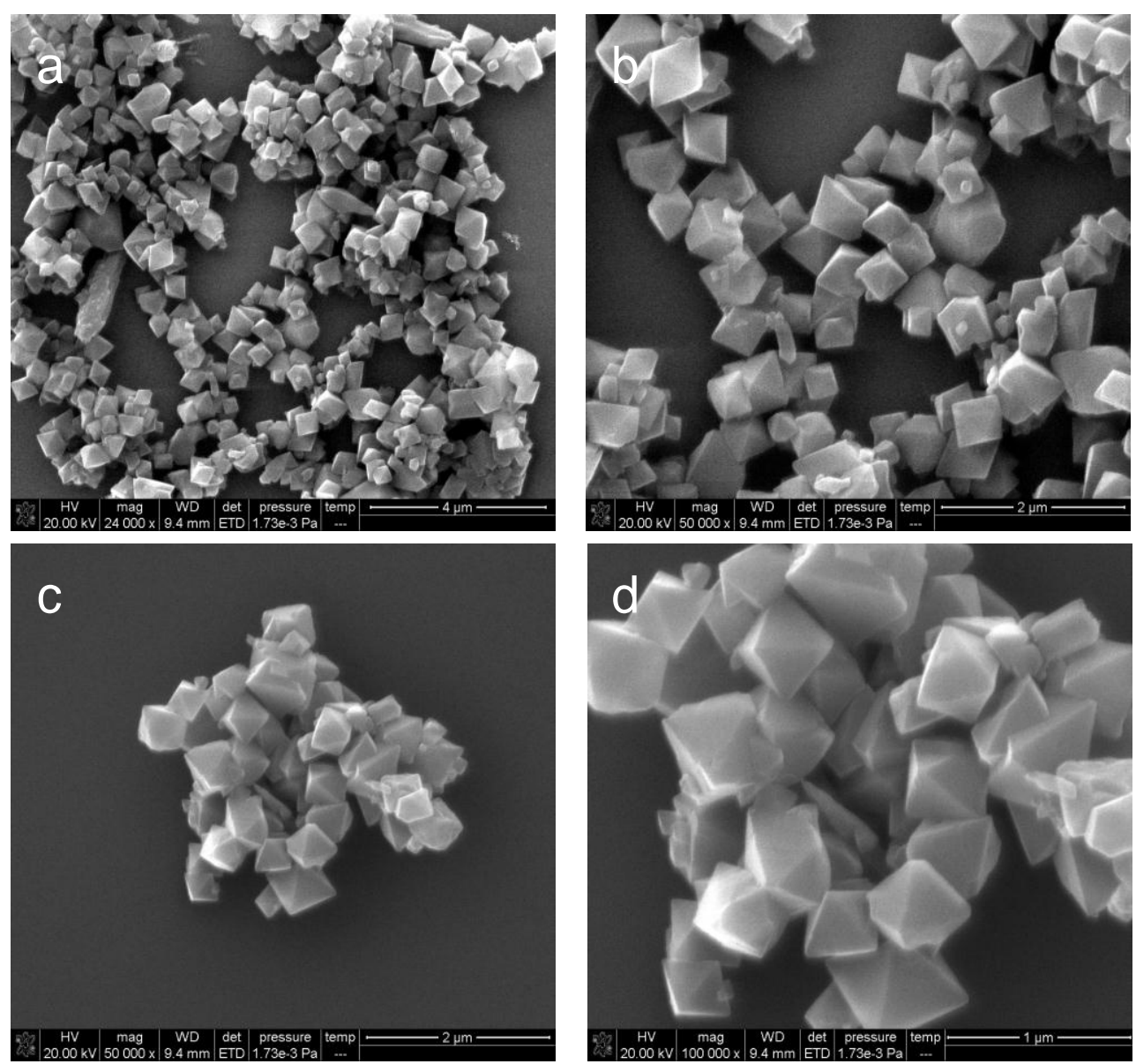

Figure 3. SEM images of MIL-101(Cr) (a,b) and MIL-101(Cr)-Cl (c,d).

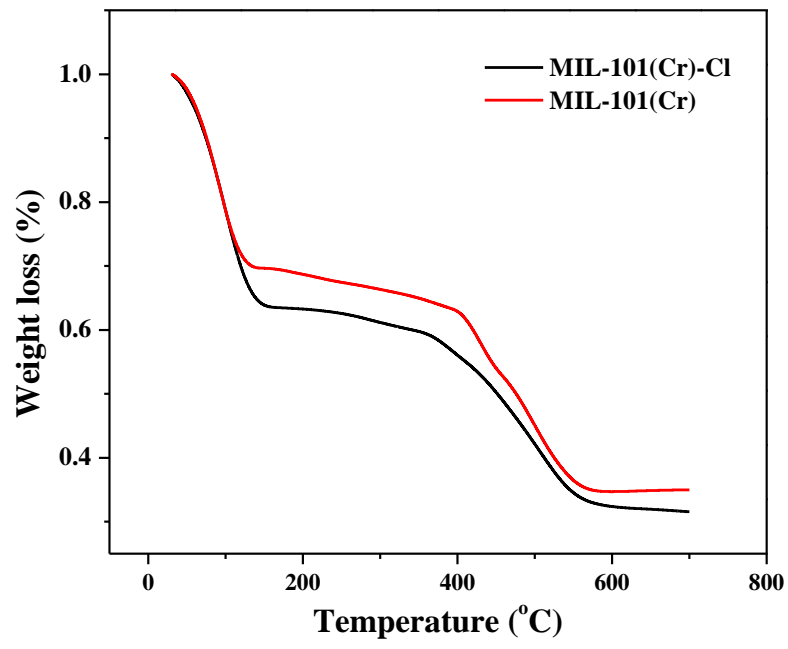

Figure 4. TGA graph of MIL-101(Cr) (red line) and MIL-101(Cr)-Cl (black line).

From Figure 5a, we can see that a white precipitate was found in the sample soaked in $80 \mathrm{mg} / \mathrm{mL}$ $\mathrm{NaNO}_{3}$, which was not found in the sample soaked in water. This phenomenon means that $\mathrm{Cl}^{-}$ has taken the place of $\mathrm{F}^{-}$and $\mathrm{Cl}^{-}$can be removed. The mobility of $\mathrm{Cl}^{-}$was further confirmed by adsorbing anionic dye molecules under the same condition. Typically, $10 \mathrm{mg}$ of MIL-101(Cr)-Cl powder was added into a $10 \mathrm{~mL}$ vial, and $10 \mathrm{~mL}$ of $50 \mathrm{mg} / \mathrm{L}$ methyl orange (MO), methylene blue, or mixed 
dye was added to the vial. After $4 \mathrm{~h}$ of adsorbing and standing, it is clear that MIL-101(Cr)-Cl is more receptive to the negatively charged $\mathrm{MO}$ (Figure $5 \mathrm{~b}$ ).
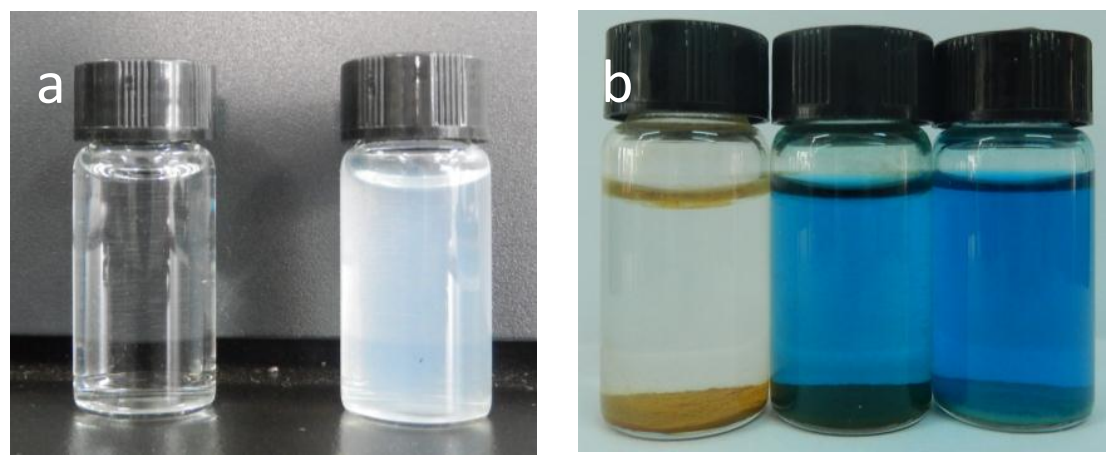

Figure 5. (a) Left is the supernatant liquid of MIL-101(Cr)-Cl soaked in water with added $\mathrm{AgNO}_{3}$ solution, right is the supernatant liquid of MIL-101(Cr)-Cl soaked in $\mathrm{NaNO}_{3}$ solution with added $\mathrm{AgNO}_{3}$ solution; (b) Left is methyl orange (MO) after adsorption by MIL-101(Cr)-Cl, middle is $\mathrm{MO}+\mathrm{MB}$ after adsorption by MIL-101(Cr)-Cl, and right is MB after adsorption by MIL-101(Cr)-Cl.

\subsection{Adsorptive Kinetics}

The kinetics of the adsorption of 2,4-D from water onto MIL-101(Cr)-Cl were studied as a function of contact time-dependent adsorption capacity. From Figure $6 a$, we can see that the adsorption capacities increased rapidly in the first $100 \mathrm{~min}$ and then slowed down; adsorption equilibrium was reached in about $200 \mathrm{~min}$. The rapid removal at the initial time could be attributed to the abundance of $\mathrm{Cl}^{-}$sites that can be removed in the MOFs and the positively charged surface of MIL-101(Cr)-Cl. With time, increasing concentration of $\mathrm{Cl}^{-}$sites and reduction in the amount of the positive charge, the adsorption rate decreased gradually at high conversion. Besides, the 2,4-D adsorbed in the MOFs at the initial time might partially block the cavities inside the MOFs, which has a negative impact on the adsorption rate.

The adsorption kinetics were simulated using a pseudo-second-order model. The pseudo-secondorder equations can be expressed as:

$$
\frac{d q}{d t}=k_{2}\left(q_{e}-q_{t}\right)^{2},
$$

or

$$
\frac{t}{q_{t}}=\frac{1}{k_{2} q_{e}^{2}}+\frac{t}{q_{e}}
$$

where $q_{e}(\mathrm{mg} / \mathrm{g})$ and $q_{t}(\mathrm{mg} / \mathrm{g})$ are the 2,4-D adsorbing capacity at equilibrium and the time $t(\mathrm{~min})$, respectively; $k_{2}(\mathrm{~g} /(\mathrm{mg} \mathrm{min}))$ is the pseudo-second-order adsorption rate constant; $k_{2}$ can be calculated from the slope and intercept of the plot of $t / q t$ versus $t$ in Figure $6 \mathrm{~b}$.

From Figure $6 \mathrm{~b}$, we can see that the correlation coefficients at different temperatures are nearly 1.00, which means that 2,4-D adsorption on MIL-101(Cr)-Cl follows the pseudo-second-order model. This model was developed based on the assumption that the limiting step may be a chemisorptions process involving valency forces via the exchange of elections between the adsorbate and adsorbent. 

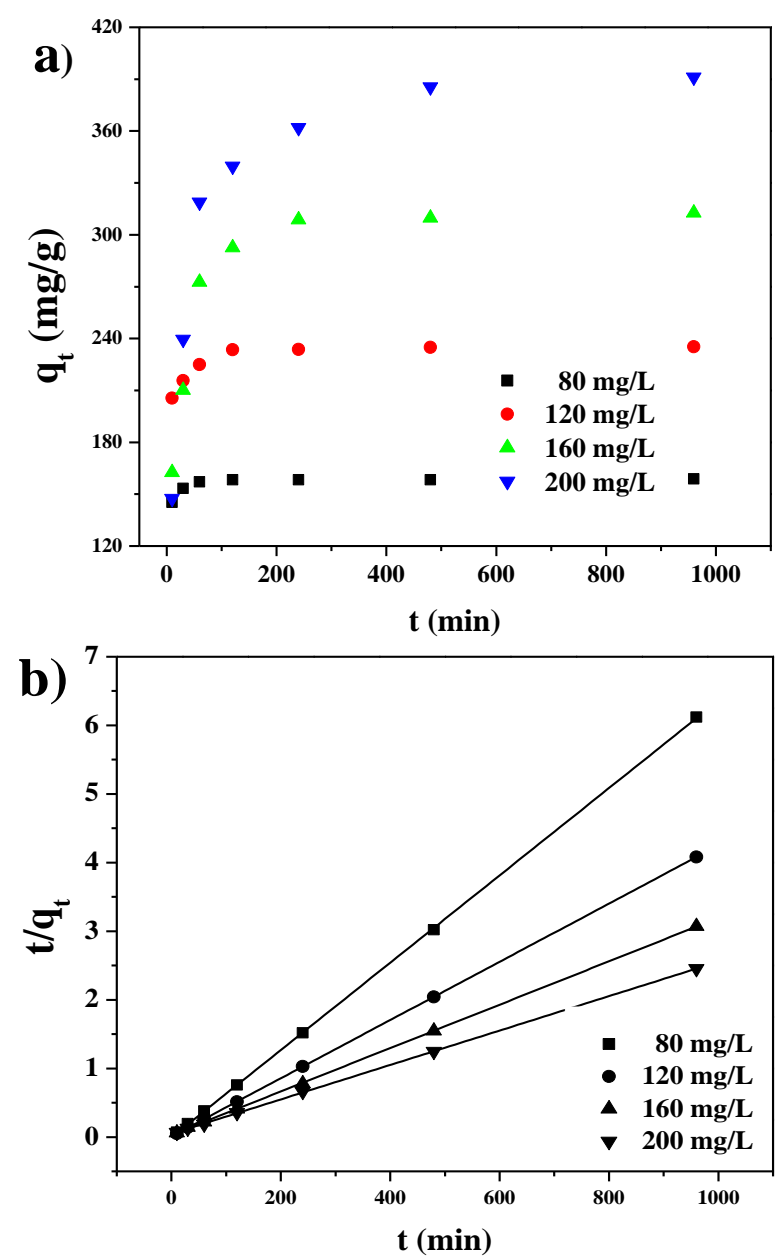

Figure 6. (a) Effect of contact time on the adsorption of 2,4-D on MIL-101(Cr)-Cl (10 mg) at different initial concentrations of 2,4-D at $30^{\circ} \mathrm{C}$; (b) Plots of pseudo-second-order kinetics for the adsorption of 2,4-D at $30^{\circ} \mathrm{C}$.

\subsection{Adsorptive Isotherms}

The adsorption isotherm model was widely used to describe the adsorption progress and investigate the mechanisms of adsorption. In this work, the adsorption isotherms of 2,4-D over MIL-101(Cr) and MIL-101(Cr)-Cl were obtained by adsorbing for $\sim 16 \mathrm{~h}$, and the results are shown in Figure 7.

From Figure 7a, it is clear that the adsorption capacities of MIL-101(Cr)-Cl were much higher than those of MIL-101(Cr) under the same test conditions, suggesting that anion stripping could enhance the efficiency of adsorbing 2,4-D.

In order to describe the adsorption isotherms of 2,4-D adsorbed over MIL-101(Cr) more clearly, the commonly used Langmuir model and three different temperatures were selected for this study [29]. The Langmuir equation can be expressed as:

$$
\frac{C_{e}}{q_{e}}=\frac{1}{q_{\max } K_{L}}+\frac{C_{e}}{q_{\max }}
$$

or

$$
q_{e}=\frac{q_{\max } K_{L} C_{0}}{1+K_{L} C_{e}}
$$

where $K_{L}(\mathrm{~L} / \mathrm{mg})$ is the Langmuir constant, and $q_{\max }(\mathrm{mg} / \mathrm{g})$ is the maximum adsorption. 
As is shown in Figure 7c, this test proved that the method conforms well to the Langmuir model. This means that this model can explain the adsorption of 2,4-D on MIL-101(Cr)-Cl. At the same time, we can see that the highest adsorption capacity is at $40^{\circ} \mathrm{C}$; we also calculated the adsorption capacities at $30^{\circ} \mathrm{C}, 40{ }^{\circ} \mathrm{C}$ and $50{ }^{\circ} \mathrm{C}$ as $416.7 \mathrm{mg} / \mathrm{g}, 497.5 \mathrm{mg} / \mathrm{g}$ and $330.3 \mathrm{mg} / \mathrm{g}$, respectively, from the slope of the line shown in Figure $7 \mathrm{~b}$.
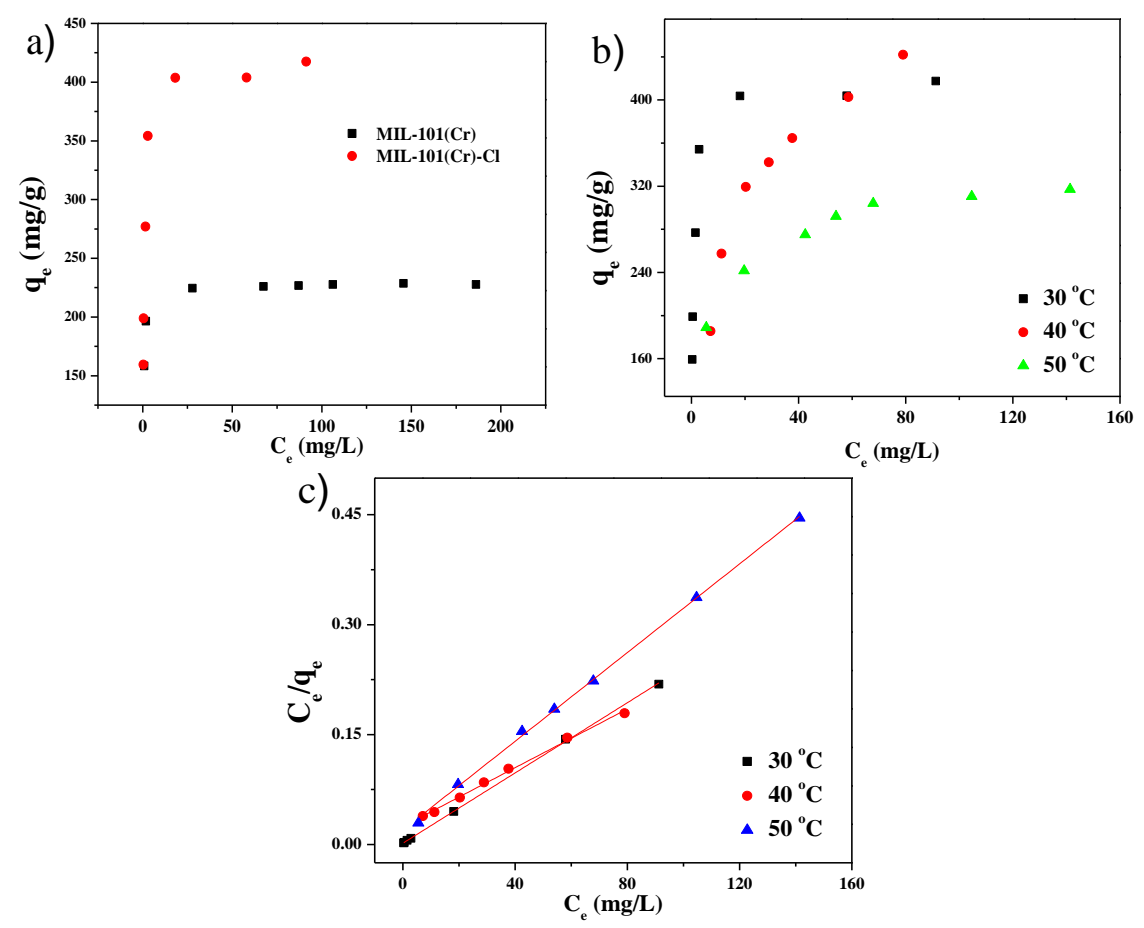

Figure 7. (a) Standard adsorption curves for 2,4-D on MIL-101(Cr) (black line) and MIL-101(Cr)-Cl (red line) at $30{ }^{\circ} \mathrm{C}$; (b) adsorption isotherms for MG on MIL-101(Cr)-Cl at different temperatures; and (c) Langmuir plots of the isotherms for the adsorption of 2,4-D adsorption onto MIL-101(Cr)-Cl.

\subsection{Adsorption Mechanism}

As shown in Figure 8a, the adsorption capacity of 2,4-D on MIL-101(Cr) decreased with increasing $\mathrm{pH}$. The low $\mathrm{pKa}$ (2.8) made it easy to ionize when the $\mathrm{pH}$ of solution is greater than 3.0. According to Figure $8 b$, the zeta potential of MIL-101(Cr) is positive when $\mathrm{pH}<6$. Hence, the repulsive electrostatic interaction between MIL-101(Cr) and 2,4-D can explain this phenomenon. The isoelectric point of MIL-101(Cr) is about 6.5, and the reason for the lower adsorption capacity was that MIL-101(Cr) and 2,4-D had positively charged surfaces. While the adsorption capacity does not reduce to zero, the lower capacity might be explained by the $\pi-\pi$ stacking of benzene rings between $2,4-\mathrm{D}$ and the organic ligands in MOFs. This result fits with previous reports $[2,25,30]$ perfectly.

Comparing the trend of the $\mathrm{pH}$ and zeta potential of $\mathrm{MIL}-101(\mathrm{Cr})$ and $\mathrm{MIL}-101(\mathrm{Cr})-\mathrm{Cl}$ (Figure $8 \mathrm{a}-\mathrm{d}$ ), it is obvious that their trends are similar. Hence, the capacity of MIL-101(Cr)-Cl should be the same as that of MIL-101(Cr), but from Figure 8c, it is clear that the capacity remained high when the $\mathrm{pH}>6$. This means that there must be another force between 2,4-D and adsorbents besides the electrostatic interaction and $\pi-\pi$ stacking. Since the $\mathrm{Cl}^{-}$in MIL-101(Cr)-Cl can be removed, it is speculated that ion exchange also occurs during the adsorption process. This phenomenon has also been experimentally verified (see the Supporting information); the precipitation experiment before and after the adsorption of 2,4-D also showed that MIL-101(Cr)-Cl had excellent performance in adsorbing negatively charged organic pollutants (Figure S3). The capacity of MIL-101(Cr)-Cl was much higher than that of MIL-101(Cr) at $\mathrm{pH}=7.0$, which is a visible sign of the ion-exchange adsorption process. Both tests proved that ion change existed during the adsorbing of 2,4-D on MIL-101(Cr)-Cl. 

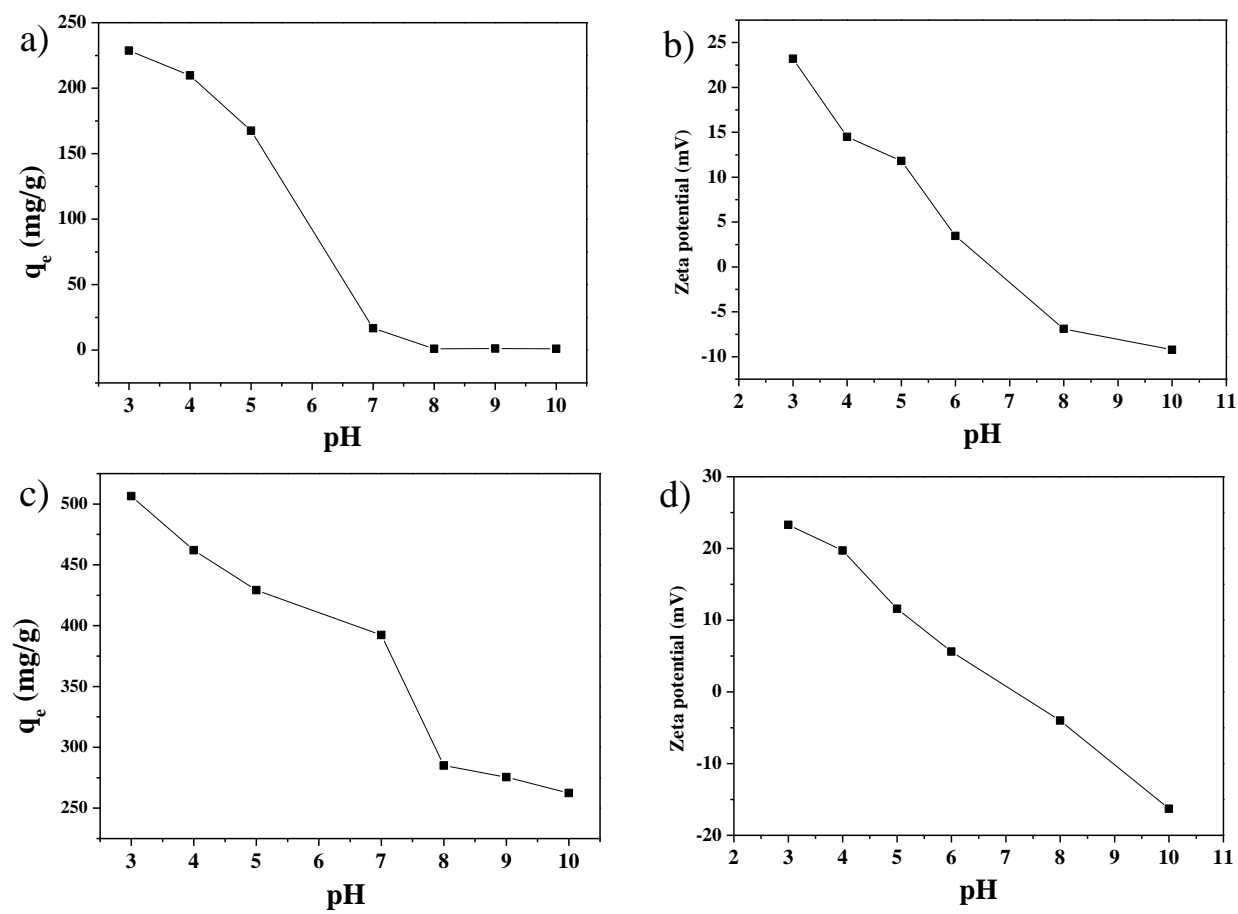

Figure 8. (a) Effect of $\mathrm{pH}$ of 2,4-D solution on the adsorption capacity of 2,4-D over MIL-101(Cr); (b) zeta potential of MIL-101(Cr) in different $\mathrm{pH}$ solutions; (c) effect of $\mathrm{pH}$ of 2,4-D solution on the adsorption capacity of 2,4-D over MIL-101(Cr)-Cl; (d) zeta potential of MIL-101(Cr)-Cl in different $\mathrm{pH}$ solutions.

\subsection{Regeneration}

From the economic and environmental viewpoints, it is necessary to reuse adsorbents. The desorption and regeneration of 2,4-D over MIL-101(Cr)-Cl were carried out by washing with $\mathrm{NaCl}-\mathrm{HCl}$ solution $(\mathrm{pH}=2.0)$ and ethanol. As can be seen from Figure 9, the adsorption capacity changed little in three cycles, which reveals that the materials can be reused.

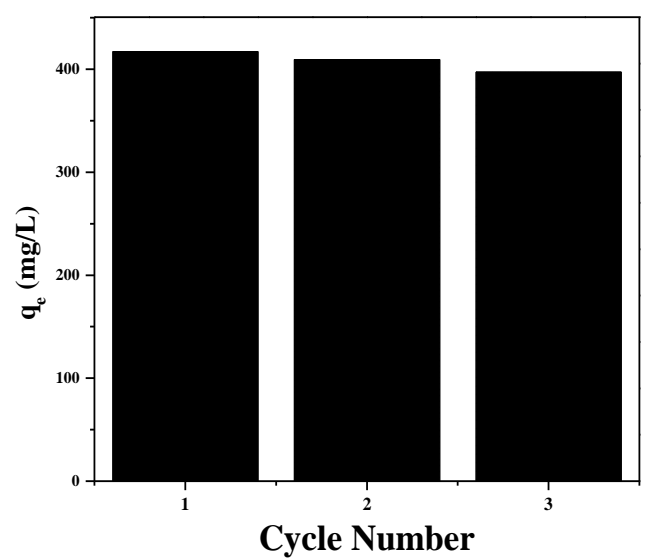

Figure 9. Eluting regeneration cycles and adsorption capacity.

\section{Materials and Methods}

\subsection{Characterization of As-Prepared Materials}

Terephthalic acid $\left(\mathrm{H}_{2} \mathrm{BDC}\right)$ and aluminum (III) chloride hexahydrate $\left(\mathrm{AlCl}_{3} \cdot 6 \mathrm{H}_{2} \mathrm{O}\right)$ were purchased from Aladdin (Shanghai, China). Chromium nitrate $\left(\mathrm{Cr}\left(\mathrm{NO}_{3}\right)_{3} \cdot 9 \mathrm{H}_{2} \mathrm{O}\right)$, ammonium fluoride 
$\left(\mathrm{NH}_{4} \mathrm{~F}\right)$, ethanol, and hydrofluoric acid ( $\left.\mathrm{HF} \geq 40 \%\right)$ were received from Shanghai Chemical Reagents Corporation (Shanghai, China). 2,4-D was purchased from Alfa Aesar (Shanghai, China). Ultrapure water was produced with a Millipore purification system (Bedford, MA, USA). All the above chemicals were at least of analytical reagent grade, and used without any purification.

\subsection{Apparatus}

Powder X-ray diffraction analysis (PXRD) was carried out using a D/max $2550 \mathrm{VB} / \mathrm{PC}$ diffractometer (Rigaku, Tokyo, Japan) operating with $\mathrm{Cu}-\mathrm{K} \alpha$ radiation $(\lambda=0.15418 \mathrm{~nm})$. The morphologies of the as-synthesized samples were characterized using field emission scanning electron microscopy (SEM; NoVaTM Nano SEM 430, FEI, Hillsboro, OR, USA). The specific surface areas and pore-size distributions were calculated by Brunauer-Emmett-Teller (BET) methods measured on 3Flex (Micromeritics, Atlanta, GA, USA) by nitrogen adsorption and desorption at $77 \mathrm{~K}$.

\subsection{Synthesis of MIL-101(Cr)}

As illustrated in Scheme 1, MIL-101(Cr) was prepared by a hydrothermal reaction previously reported by Férey and co-workers [31]. Typically, $166 \mathrm{mg}$ of $\mathrm{H}_{2} \mathrm{BDC}, 400 \mathrm{mg}$ of $\mathrm{Cr}\left(\mathrm{NO}_{3}\right)_{3} \cdot 9 \mathrm{H}_{2} \mathrm{O}$, and $1 \mathrm{mmol} \mathrm{HF}$ were dissolved in $4.8 \mathrm{~mL}$ of water, and then loaded into a Teflon-lined autoclave at $220^{\circ} \mathrm{C}$ for $8 \mathrm{~h}$. We obtained a highly crystallized green powder (original MIL-101(Cr)). However, the unreacted $\mathrm{Cr}(\mathrm{III})$ and $\mathrm{H}_{2} \mathrm{BDC}$ are present both within and outside the pores of MIL-101(Cr). Therefore, it is necessary to further purify the as-synthesized MOFs. The purification method comprises the following steps: (1) the crystalline MIL-101(Cr) product was filtered after the reaction system cooled slowly to room temperature, and then washed with a large amount of hot water to remove the unreacted $\mathrm{Cr}$ (III) and $\mathrm{H}_{2} \mathrm{BDC}$ on its surface; (2) the resulting solid was soaked in $30 \mathrm{mmol} \mathrm{NH}_{4} \mathrm{~F}$ solution at $80{ }^{\circ} \mathrm{C}$, stirred for $24 \mathrm{~h}$ in order to swap out the unreacted $\mathrm{Cr}(\mathrm{III})$ and $\mathrm{H}_{2} \mathrm{BDC}$ within the pores of MIL-101(Cr), and immediately filtered; (3) the residue was washed with a certain amount of water and ethanol. The solid was finally dried at $150{ }^{\circ} \mathrm{C}$ for $24 \mathrm{~h}$ under vacuum.

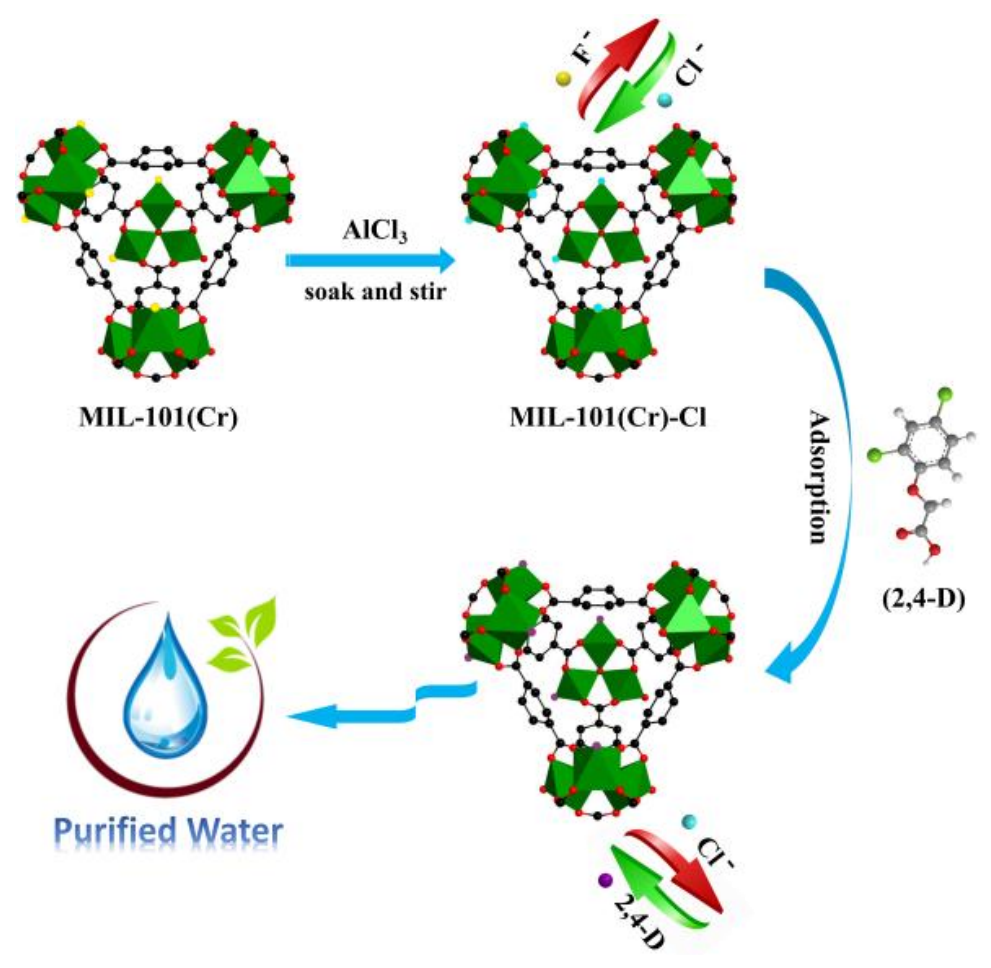

Scheme 1. Synthesis of MIL-101(Cr)-Cl. 


\subsection{Anion Stripping of MIL-101(Cr)}

To the as-synthesized MIL-101(Cr) $(50 \mathrm{mg})$ in a $100 \mathrm{~mL}$ vial was added $50 \mathrm{~mL} 30 \mathrm{mmol}$ of $\mathrm{AlCl}_{3}$ solution, and then the vial was shifted into a $90^{\circ} \mathrm{C}$ oven and kept for about $\sim 16 \mathrm{~h}$. The final material (denoted MIL-101(Cr)-Cl) was obtained by centrifugation and washed with water until the effluents did not turn $\mathrm{AgNO}_{3}$ turbid. The as-prepared material was degassed under vacuum at $150{ }^{\circ} \mathrm{C}$ overnight before use.

\subsection{Adsorption Procedure}

The working solution of 2,4-D used in this test was prepared by the dilution of a stock solution (300 mg/L), which was prepared by dissolving powdered 2,4-D in deionized water. Before adsorption, the as-prepared materials, MIL-101(Cr) and MIL-101(Cr)-Cl, were degassed and dried under vacuum at $150{ }^{\circ} \mathrm{C}$ overnight. To an exact amount of degassed and dried as-prepared materials $(5 \mathrm{mg})$ placed in a $20 \mathrm{~mL}$ vessel, was added $10 \mathrm{~mL}$ of 2,4-D solution. The vessel was fixed on a vortex generator and placed into a water bath. After a pre-determined adsorption time, the suspensions were separated using a syringe filter. The concentrations of 2,4-D in the solution were determined using a spectrophotometer (Agilent, Shanghai, China) at $283 \mathrm{~nm}$ after taking the UV spectra of the solution. The standard curve of 2,4-D (Figure S4) was obtained from standard solutions $(5-200 \mathrm{mg} / \mathrm{L}$ ) at $\mathrm{pH}=3.5$.

The adsorption capacity at equilibrium was calculated as follows:

$$
q_{e}=\frac{C_{0}-C_{e}}{M} V
$$

where $q_{e}(\mathrm{mg} / \mathrm{g})$ is the equilibrium adsorption capacity of 2,4-D on the as-prepared material; $C_{0}(\mathrm{mg} / \mathrm{L})$ and $C_{e}(\mathrm{mg} / \mathrm{L})$ are the initial and equilibrium concentrations of 2,4-D, respectively; $V$ is the volume of 2,4-D solution (L); and $M$ is the weight of the as-prepared material (g).

\section{Conclusions}

In summary, we successfully synthesized MIL-101(Cr) and MIL-101(Cr)-Cl by a simple hot $\mathrm{AlCl}_{3}$ solution treatment step. Both of them were used as adsorbents for removing 2,4-D. The experimental results show that after modification, MIL-101(Cr) has a higher capacity and the capacity remained high at high $\mathrm{pH}$. While studying the adsorption mechanism, we found that in addition to electrostatic interaction and $\pi-\pi$ stacking, ion exchange might also play a large part in the adsorption process. Hence, MIL-101(Cr)-Cl may have a great potential in adsorbing negatively charged organic pollutants, and this method of exchanging $\mathrm{F}^{-}$with $\mathrm{Cl}^{-}$in the original MOFs could be generalized to generate new charged MOFs.

Supplementary Materials: The following are available online at www.mdpi.com/1996-1944/10/8/879/s1, Figure S1: (a) The BJH pore size of MIL-101(Cr); (b) The HK pore size of MIL-101(Cr). Figure S2: (a) The BJH pore size of MIL-101(Cr)-Cl; (b) The HK pore size of MIL-101(Cr)-Cl. Figure S3: The photos of (a) $100 \mathrm{mg} / \mathrm{L}$ of 2,4-D added $\mathrm{AgNO}_{3}$ solution; (b) after adsorbed 2,4-D, the supernatants added $\mathrm{AgNO}_{3}$ solution. Figure S4: The calibration curve of 2,4-D, $A=0.0085 C+0.0184, R^{2}=0.999$. Table S1: The fluorine and chlorine content in MIL-100-Cr and MIL-100-Cr-Cl.

Acknowledgments: The financial support from the National Natural Science Foundation of China (21365005) and Guangxi Natural Science Foundation of China (2014GXNSFGA118002) is gratefully acknowledged.

Author Contributions: Fanggui Ye conceived and designed the experiments; Cong Zhang performed the experiments; Yuemei Qin analyzed the data; Haiguan Yang and Peng Zhang contributed reagents and analysis tools; Tao Chen wrote the paper.

Conflicts of Interest: The authors declare no conflict of interest.

\section{References}

1. Ahmed, A.B.; Jibril, B.; Danwittayakulb, S.; Dutta, J. Microwave-enhanced degradation of phenol over Ni-loaded ZnO nanorods catalyst. Appl. Catal. B Environ. 2014, 156, 456-465. [CrossRef] 
2. Daneshvar, N.; Khataee, A.R.; Rasoulifard, M.H.; Pourhassan, M. Biodegradation of dye solution containing Malachite Green: Optimization of effective parameters using Taguchi method. J. Hazard. Mater. 2007, 143, 214-219. [CrossRef] [PubMed]

3. Wei, F.; Cao, C.; Huang, P.; Song, W. A new ion exchange adsorption mechanism between carbonate groups and fluoride ions of basic aluminum carbonate nanospheres. RSC Adv. 2015, 5, 13256-13260. [CrossRef]

4. Zhang, S.; Zeng, M.; Li, J.; Li, J.; Xu, J.; Wang, X. Porous magnetic carbon sheets from biomass as an adsorbent for the fast removal of organic pollutants from aqueous solution. J. Mater. Chem. A 2014, 2, 4391-4397. [CrossRef]

5. Nakamura, H.; Okumura, M.; Machida, M. Monte Carlo simulation studies of cation selectivity in ion exchange of zeolites. RSC Adv. 2014, 4, 52757-52761. [CrossRef]

6. Peng, F.; Luo, T.; Yuan, Y. Controllable synthesis of Mg-Fe layered double hydroxide nanoplates with specific $\mathrm{Mg} / \mathrm{Fe}$ ratios and their effect on adsorption of $\mathrm{As}(\mathrm{V})$ from water. New J. Chem. 2014, 38, 4427-4433. [CrossRef]

7. Moya, A.A. Theory of the formation of the electric double layer at the ion exchange membrane-solution interface. Phys. Chem. Chem. Phys. 2015, 17, 5207-5218. [CrossRef] [PubMed]

8. Prakash, S.; Kumar, M.; Tripathi, B.P.; Shahi, V.K. Sol-gel derived poly (vinylalcohol)-3-(2-aminoethylamino) propyl trimethoxysilane: Cross-linked organic-inorganic hybrid beads for the removal of $\mathrm{Pb}$ (II) from aqueous solution. Chem. Eng. J. 2010, 162, 28-36. [CrossRef]

9. Chen, J.-J.; Chen, Y.-T.; Raja, D.S.; Kang, Y.-H.; Tseng, P.-C. Metal-Organic Frameworks to Metal/Metal Oxide Embedded Carbon Matrix: Synthesis, Characterization and Gas Sorption Properties. Materials 2015, 8, 5336-5347. [CrossRef]

10. García, E.R.; Medina, R.L.; Lozano, M.M.; Pérez, I.H.; Valero, M.J.; Franco, A.M.M. Adsorption of Azo-Dye Orange II from Aqueous Solutions Using a Metal-Organic Framework Material: Iron-Benzenetricarboxylate. Materials 2014, 7, 8037-8057. [CrossRef]

11. Zhang, X.L.; Zhan, Z.B.; Li, Z.; Di, L.B. Thermal Activation of CuBTC MOF for CO Oxidation: The Effect of Activation Atmosphere. Catalysts 2017, 7, 106. [CrossRef]

12. Hu, Q.; Yu, J.; Liu, M.; Liu, A.; Dou, Z.; Yang, Y. A low cytotoxic cationic metal-organic framework carrier for controllable drug release. J. Med. Chem. 2014, 57, 5679-5685. [CrossRef] [PubMed]

13. Campbell, M.G.; Dincă, M. Metal-organic frameworks as Active Materials in Electronic Sensor Devices. Sensors 2017, 17, 1108. [CrossRef] [PubMed]

14. Fernandes, D.M.; Barbosa, A.D.S.; Pires, J.; Balula, S.S.; Cunha-Silva, L.; Freire, C. Novel composite material polyoxovanadate@MIL-101(Cr): A highly efficient electrocatalyst for ascorbic acid oxidation. ACS Appl. Mater. Interfaces 2013, 5, 13382-13390. [CrossRef] [PubMed]

15. Fu, H.R.; Xu, Z.X.; Zhang, J. Water-stable metal-organic frameworks for fast and high dichromate trapping via single-crystal-to-single-crystal ion exchange. Chem. Mater. 2014, 27, 205-210. [CrossRef]

16. Tan, Y.X.; Zhang, Y.; He, Y.P.; Zheng, Y.J.; Zhang, J. Multifunctional anionic MOF material for dye enrichment and selective sorption of $\mathrm{C}_{2}$ hydrocarbons over methane via $\mathrm{Ag}^{+}$-Exchange. Inorg. Chem. 2014, 53, 12973-12976. [CrossRef] [PubMed]

17. Li, Z.J.; Khani, S.K.; Akhbari, K.; Morsali, A.; Retailleau, P. Achieve to easier opening of channels in anionic nanoporous Metal-organic framework by cation exchange process. Microporous Mesoporous Mater. 2014, 199, 93-98. [CrossRef]

18. Nanthamathee, C.; Ling, S.; Slater, B.; Attfield, M.P. Contradistinct thermoresponsive behavior of isostructural MIL-53 type Metal-organic frameworks by modifying the framework inorganic anion. Chem. Mater. 2015, 27, 85-95. [CrossRef]

19. Ding, L.; Lu, X.; Deng, H.; Zhang, X. Adsorptive removal of 2,4-dichlorophenoxyacetic acid (2,4-D) from aqueous solutions using MIEX resin. Ind. Eng. Chem. Res. 2012, 51, 11226-11235. [CrossRef]

20. Bakhtiary, S.; Shirvani, M.; Shariatmadari, H. Adsorption-desorption behavior of 2,4-D on NCP-modified bentonite and zeolite: Implications for slow-release herbicide formulations. Chemosphere 2013, 90, 699-705. [CrossRef] [PubMed]

21. Njoku, V.O.; Foo, K.Y.; Hameed, B.H. Microwave-assisted preparation of pumpkin seed hull activated carbon and its application for the adsorptive removal of 2,4-dichlorophenoxyacetic acid. Chem. Eng. J. 2013, 215, 383-388. [CrossRef]

22. Nejati, K.; Davary, S.; Saati, M. Study of 2,4-dichlorophenoxyacetic acid (2,4-D) removal by Cu-Fe-layered double hydroxide from aqueous solution. Appl. Surf. Sci. 2013, 280, 67-73. [CrossRef] 
23. Zhong, S.; Zhou, C.; Zhang, X.; Zhou, H.; Li, H.; Zhu, X.; Wang, Y. A novel molecularly imprinted material based on magnetic halloysite nanotubes for rapid enrichment of 2,4-dichlorophenoxyacetic acid in water. J. Hazard. Mater. 2014, 276, 58-65. [CrossRef] [PubMed]

24. Yang, L.; Sun, W.; Luo, S.; Luo, Y. White fungus-like mesoporous $\mathrm{Bi}_{2} \mathrm{~S}_{3}$ ball $/ \mathrm{TiO}_{2}$ heterojunction with high photocatalytic efficiency in purifying 2,4-dichlorophenoxyacetic acid/Cr(VI) contaminated water. Appl. Catal. $B$ Environ. 2014, 156, 25-34. [CrossRef]

25. Jung, B.K.; Hasan, Z.; Jhung, S.H. Adsorptive removal of 2,4-dichlorophenoxyacetic acid (2,4-D) from water with a metal-organic framework. Chem. Eng. J. 2013, 234, 99-105. [CrossRef]

26. Bhardwaj, D.; Sharma, P.; Sharma, M.; Tomar, R. Surfactant modified tectosilicates and phyllosilicates for 2,4-D removal and slow release formulation. RSC Adv. 2014, 4, 4504-4514. [CrossRef]

27. Hong, D.Y.; Hwang, Y.K.; Serre, C.; Férey, G.; Chang, J.-S. Porous chromium terephthalate MIL-101 with coordinatively unsaturated sites: Surface functionalization, encapsulation, sorption and catalysis. Adv. Funct. Mater. 2009, 19, 1537-1552. [CrossRef]

28. Mao, C.; Kudla, R.A.; Zuo, F.; Zhao, X.; Mueller, L.J.; Bu, X.; Feng, P. Anion stripping as a general method to create cationic porous framework with mobile anions. J. Am. Chem. Soc. 2014, 136, 7579-7582. [CrossRef] [PubMed]

29. Huo, S.; Yan, X. Metal-organic framework MIL-100(Fe) for the adsorption of malachite green from aqueous solution. J. Mater. Chem. 2012, 22, 7449-7455. [CrossRef]

30. Hasan, Z.; Jeon, J.; Jhung, S.H. Adsorptive removal of naproxen and clofibric acid from water using metal-organic frameworks. J. Hazard. Mater. 2012, 209, 151-157. [CrossRef] [PubMed]

31. Liu, S.; Zhang, Y.; Meng, Y.; Gao, F.; Jiao, S.; Ke, Y. Fast syntheses of MOFs using nanosized zeolite crystal seeds in situ generated from microsized zeolites. Cryst. Growth Des. 2013, 13, 2697-2702. [CrossRef]

(C) 2017 by the authors. Licensee MDPI, Basel, Switzerland. This article is an open access article distributed under the terms and conditions of the Creative Commons Attribution (CC BY) license (http:/ / creativecommons.org/licenses/by/4.0/). 